\title{
Mathematical Model of Infectious Disease with Multistage Vaccine
}

\author{
Assefa Erba Bikila, \\ College of Natural and Computational Science, Department of Mathematics, \\ Wolaita Sodo University, Sodo ,Ethiopia P.O.BOX 138 \\ Email: asebikila@gmail.com
}

\begin{abstract}
Many diseases, such as the seasonal influenza, tetanus, and smallpox, can be vaccinated against with a single dose of a vaccine. However, some diseases require multiple doses of a vaccine for immunity. Diseases requiring a multistage vaccine such as Hepatitis B can have extra complications with its vaccination program, as some who start the doses may forget to complete the program or could become infected before completing the program. This thesis concerns the setup and analysis of a model for developing a mathematical model to describe the dynamics of an infectious disease with a multistage vaccine. In this thesis, we considered Susceptible-Infected-Removed (SIR) epidemic models and discussed the mathematical analysis and simulation study is conducted. We discuss an epidemic model which represents the direct transmission of infectious disease. The model assumes that individuals are equally likely to be infected by the infectious individuals in a case of contact except those who are immune. We formulated SIR epidemiological model to determine the transmission disease by using compartmental model approach to using a system of nonlinear differential equations. We study about basic reproduction number and equilibrium point for compartmental mathematical models of infectious disease transmission. The basic reproduction number $R_{0}$, which is a threshold quantity for the stability of equilibrium point is calculated. If $R_{0}<1$ then the disease-free equilibrium point is globally asymptotically stable and it is the only equilibrium point. On the contrary, if $R_{0}>1$ then an endemic equilibrium point appears which is locally asymptotically stable.
\end{abstract}

Keywords: Equilibrium Stability, SIR, Multistage Vaccine and Basic Reproduction Number DOI: 10.7176/MTM/9-10-04

Publication date: October $31^{\text {st }} 2019$

\section{INTRODUCTION}

Disease has played an important role in the history of mankind. The spread of communicable diseases has influenced the growth of populations and economies of countries. Most people have attributed the main causes of transmission of infectious disease due to lack of proper education about the infectious disease and low detection rate at early stages, financial constrains is also a major factor as most people prefer the treatment of the disease locally or traditionally than exposing themselves to hospitals treatment.

In 2009, a novel strain of the $\mathrm{H}_{1} \mathrm{~N}_{1}$ influenza virus, which was referred to as the "Swine Flu" in the mainstream media, caused a worldwide pandemic, eventually causing about 18,500 laboratory-confirmed deaths worldwide (the estimated number of deaths worldwide was around 285,000) (Dawood 2012). Luckily, the $2009 \mathrm{H}_{1} \mathrm{~N}_{1}$ 
pandemic did not turn out to be as devastating as, say, the 1918-1919 Spanish Flu pandemic, which killed millions of people worldwide. In the summer of 2009, a vaccine against the virus was eventually developed. However, studies indicated that children between the ages of 6 months and 9 years would need to receive two doses of the vaccine, spread about 28 days apart, in order to be fully immunized (Centres for Disease Control and Prevention 2009).

Hepatitis B is an infection of the liver caused by the hepatitis B virus (HBV). It is a globally infectious disease. According to World Health Organization, about 350 million people are infected with the hepatitis B (WHO, 2008). Facts indicate that hepatitis B virus is liable for almost 80 percent of the primary liver cancer, which is one of the most common tumours in man.

The most successful way of controlling infectious disease is vaccination. In China, hepatitis B virus infection is a major public health problem. So, hepatitis B, need three doses of the vaccine for immunity. The second one occurs about 1 month after the first, and the third occurs about 5 months after the second (Centres for Disease Control and Prevention 2012). This could cause a number of issues with immunizing a population. People may get sick before finishing the doses, or people may forget to receive future doses in the recommended time frame, rendering the already received doses effectively wasted. If this happens, then the vaccine supply, which may already be stressed by people needing to receive multiple doses, may end up not immunizing enough people to wipe out the disease.

Models for infectious diseases are helpful for prevention and control of emerging infectious diseases. The Kermack and McKendrick model is a compartmental model based on relatively simple assumptions on the rates of flow between different classes of numbers of the population (Kermack, W.O. and A.G. McKendrick, 1932). An important result concerning the idea of epidemic thresholds was published in 1927 by Kermack and McKendrick. An epidemic represents the sudden outbreak of a disease often occurring on a short temporal scale and affecting a significant portion of a population.

Thus, we set out to formulate and analyze a mathematical model of a population with an infectious disease spreading through it that required a multistage vaccine in order to be fully immunized. An epidemic model is a simplified means of describing the transmission of communicable disease through individuals. The progress of individuals is schematically described by $\mathrm{S} \rightarrow \mathrm{I} \rightarrow R$, these types of models are known as SIR models in general. The SIR model is used in epidemiology to compute the amount of susceptible (S), infected (I) and recovered (R) population. This mathematical model was inspired by the classic SIR epidemic model by Kermack and McKendrick in 1927 and also by Andrei Korobeinikov's 2009 work, which split the infectious group into multiple subgroups based on their likelihood of spreading the infection (Korobeinikov 2009). Infectious diseases that target children include chicken pox, smallpox, measles and whooping cough (Hethcote, 2000). Measles is one of the most infectious diseases and has severe complications, including inflammation of the brain and death.

Demirci and Unal (2010), introduced a fractional order SEIR epidemic model with vertical transmission, where the death rate of the population is density dependent, i.e., dependent on the population size. It is also assumed 
that there exists an infection related death rate. They also show the existence of nonnegative solutions of the model, and also give a detailed stability analysis of disease free and positive fixed points.

\section{Design and Methodology}

A research needs a firm design or structure before commencement of data collection and general research process for the functions of enabling the researcher in order to obtained evidences answer the initial research in unambiguous way. Therefore, this research work was undertaken with design of the study instruments.

We develop a mathematical model which describes the dynamics of infectious disease and its transmission using a system of non-linear ordinary differential equations. The analysis of the model was done using different tools. The method used in this study divides the population into three compartments (S, I, R) consisting of susceptible, Infected and Recovered class. Determination of equilibrium points and their stability is then performed as well as basic reproduction number is found. The Jacobean matrix is used to find sufficient conditions for the local stability of the equilibrium points of the system in the model equations. The Lyapunov function is used to show global stability of a positive equilibrium point of the system of the model equations. To supplement the analytical solutions of the model equations we used MATLAB and mathematica for the numerical simulations.

\section{MODEL FORMULATION AND ANALYSIS}

To formulate our model, we first assume that the disease we are studying will break up our population into three groups. These are susceptible (S) those individuals who are healthy and can be infected, infective (I) those individuals who are infected and are able to transmit the diseases, and recovered $(\mathrm{R})$ those individuals who are immune because have been infected and now have recovered.

The possible flow of individuals from one group to another is $S \rightarrow I \rightarrow R$. The SIR model is used for modeling general epidemics and to know how the spread of a disease is in a particular population and some possible ways of controlling such a disease. This description of the SIR model was made more mathematical by a formulating differential equation for the rate of proportion of individuals in each class.

To use the classic Kermack and McKendrick formulation of the SIR model, we further assume that the population we are studying is relatively small (say, a small, relatively isolated town), where every contact between two randomly selected individuals is equally likely.

The infectious diseases spread from an infected individual to other susceptible individuals in the surroundings. There is no treatment failure, a patient will either recover or die. The total population at time t represented by $\mathrm{N}(\mathrm{t})$ is considered as a constant and is the sum of the populations in the compartments $\mathrm{S}(\mathrm{t}), \mathrm{I}(\mathrm{t})$ and $\mathrm{R}(\mathrm{t})$, that is $\mathrm{N}$ $=\mathrm{S}+\mathrm{I}+\mathrm{R}$. The population is closed in the sense that the immigrations, new births and deaths of people are not considered.

Here we consider SIR mathematical model assuming that the numbers of both births and deaths are equal since the time duration under consideration is quite short and as a result population size remains to be a constant 
during this period of time. Infectious individuals are detected early and isolated for immediate treatment and education.

Every person in our population is susceptible to the infectious disease. The population is homogeneously mixed. We will also assume that the per capita infection rate as well as the rate of recovery remains constant. We then introduce a multistage vaccine that can result in susceptible becoming immune. Hence, those who complete the vaccination program before becoming sick can go right into the recovered group. For this model, we will assume that those who receive immunity via vaccination will be placed in a separate group, $\mathrm{V}$.

To administer the vaccine, we initially assume that a proportion of individuals will embark on the vaccination program, and others will choose not to. After administering these first doses (this will be built into the initial conditions for the model), we specify that any subsequent dose must occur at a time $\tau_{i}, \mathrm{i}=1,2 \ldots \mathrm{n}-1$ after the first dose is administered. However, practically it is not feasible to administer all of the vaccines during a single instant of time, and so we will also allow a grace period to receive each vaccine. Thus, $\tau_{i}$ represents the deadline upon which an individual must receive the $\mathfrak{i}^{\text {th }}$ dose, and there is an interval of length $g_{i}$ that ends at $\tau_{i}$ such that someone may receive that dose any time in that interval. Specifically, if we refer to the example of the 2009 $\mathrm{H}_{1} \mathrm{~N}_{1}$ influenza vaccine (inactivated virus) for those children aged 6 months to 9 years, the second dose should be given 28 days after the first dose, but can potentially be given as early as 21 days after the first dose (Centers for Disease Control and Prevention 2009). So, this would allow an individual to receive the second dose any time between days 21 and 28 , with $\tau_{1}=28$ days and $g_{1}=7$ days. We also assume that the grace periods do not overlap; this is to prevent someone from potentially receiving two doses at the same time, and for a clear distinction as to who should be in which group at a given time.

Next, we can incorporate births and deaths into the population. We specifically say that the birth rate and the per capita death rate are constant, and that all of the births are assumed susceptible. Thus, the births would be placed into the $\mathrm{S}$ group, with the per capita death rate being the same for all groups.

Finally, for this first formulation of the model, we will only administer the full vaccine, or all $\mathrm{n}$ doses, once. Thus, we will assume that those who do not complete the vaccination program, but do not get sick, will simply remain in which ever susceptible group represents how many total doses they received unless they become sick.

Based on these assumptions, we define the following variables and parameters

$\mathrm{S}(\mathrm{t})$ Susceptible individuals at time t,

$\mathrm{I}(\mathrm{t})$ Infective at time $\mathrm{t}$,

$\mathrm{R}(\mathrm{t})$ Recovered individuals at a time $\mathrm{t}$,

$\mathrm{V}(\mathrm{t})$ Vaccinated at time $\mathrm{t}$,

b Per capita infection rate 
a Recovery rate; $1 / \mathfrak{a}$ represents the average time an individual is infective

$N_{0}$ Initial population size

$\beta$ Birth rate

$\mu$ Per capita death rate

$\rho$ Proportion of those successively vaccinated at birth

This then leads us to the following system of first order nonlinear differential equations for our model

$$
\begin{gathered}
\frac{d S}{d t}=\beta-b S \mathrm{I}-\mu S \\
\frac{d I}{d t}=b S \mathrm{I}-\mathfrak{a} \mathrm{I}-\mu \mathrm{I} \\
\frac{d R}{d t}=\mathfrak{a I}-\mu \mathrm{R} \\
\frac{d V}{d t}=\rho-\mu \mathrm{V} \\
\frac{d N}{d t}=0 \text { and } \mathrm{N}=\mathrm{S}+\mathrm{I}+\mathrm{R} \text { is a constant }
\end{gathered}
$$

Lemma 1(Positivity solutions): If the initial data set be (S, I, R, V) $(0) \geq 0 \epsilon \Omega$, then the solution set (S, I, R, V) (t) of the system of equations in $(1-4)$ is positive for all $t>0$, where $\Omega=\{\mathrm{S}, \mathrm{I}, \mathrm{R}, \mathrm{V}\}$.

Proof: To determine the positivity of $\mathrm{S}$, we consider

$$
\begin{aligned}
& \frac{\mathrm{dS}}{\mathrm{dt}}=-\mathrm{bSI}+\beta-\mu \mathrm{S} \leq(\beta-\mu \mathrm{S}) \\
& \frac{\mathrm{dS}}{\mathrm{dt}} \leq(\beta-\mu \mathrm{S}) \\
& \frac{\mathrm{dS}}{\mathrm{dt}}+\mu \mathrm{S} \leq \beta
\end{aligned}
$$

By computing the integrating factor $\mathrm{F}(\mathrm{t})=e^{\mu t}$, we obtained

$$
\begin{aligned}
& e^{\mu t}\left(\frac{d S}{d t}+\mu S \leq \beta\right) \\
& \frac{d}{d t}\left(S e^{\mu t}\right) \leq \beta e^{\mu t}
\end{aligned}
$$

Integrating both side of the inequalities, gives

$$
\mathrm{S}(\mathrm{t}) \leq \frac{\beta}{\mu}+\left(\mathrm{S}(0)-\frac{\beta}{\mu}\right) e^{-\mu t}
$$

Since, $\mu>0$ and $\mathrm{S}(0) \geq 0$ then $\mathrm{S}(\mathrm{t}) \geq 0, \forall t \geq 0$. 
From equation (2),

$$
\begin{aligned}
& \frac{\mathrm{dI}}{\mathrm{dt}}=b S \mathrm{I}-(\mathfrak{a}+\mu) \mathrm{I} \geq-(\mathfrak{a}+\mu) I \\
& \frac{\mathrm{dI}}{\mathrm{dt}} \geq-(\mathfrak{a}+\mu) \mathrm{I} \\
& \frac{\mathrm{dI}}{\mathrm{I}} \geq-(\mathfrak{a}+\mu) d t
\end{aligned}
$$

Integrating both sides of the inequalities, gives

$$
\mathrm{I}(\mathrm{t}) \geq \mathrm{I}(0) e^{-(\mathfrak{a}+\mu) t} \geq 0 \text {, and } \mathrm{I}(0) \geq 0, \text { then } \mathrm{I}(\mathrm{t}) \geq 0, \forall t \geq 0
$$

From equation (3)

$$
\begin{aligned}
& \frac{d R}{d t}=\mathfrak{a I}-\mu \mathrm{R} \geq-\mu \mathrm{R} \\
& \frac{d R}{R} \geq-\mu d t
\end{aligned}
$$

Integrating both sides of the inequalities,

Thus, $\mathrm{R}(\mathrm{t}) \geq \mathrm{R}(0) e^{-\mu t} \geq 0$ and $\mathrm{R}(0) \geq 0$, then $\mathrm{R}(\mathrm{t}) \geq 0 \forall t \geq 0$

From equation (4)

$$
\begin{gathered}
\frac{d V}{d t}=\rho-\mu \mathrm{V} \geq-\mu \mathrm{V} \\
\frac{d V}{d t} \geq-\mu \mathrm{V}
\end{gathered}
$$

Integrating both sides of the inequalities, gives

Thus, $\mathrm{V}(\mathrm{t}) \geq \mathrm{V}(0) e^{-\mu t}$ and $\mathrm{V}(0) \geq 0$, then $\mathrm{V}(\mathrm{t}) \geq 0, \forall t \geq 0$.

Hence, all variables are positive for all time $\mathrm{t}>0$.

\section{Boundedness of solution}

In this section, we show a description of some basic properties of the model equations, such as feasible solution. The feasible solution shows the region in which the solutions of the equations of the system are biologically meaningful.

Lemma 2: The feasible solution set which is positively invariant set of the system of equations $(1-3)$ is given by:- 
$\Omega=\left\{(\mathrm{S}, \mathrm{I}, \mathrm{R}) \in R_{+}^{3}: S+I+R=N \leq \frac{\beta}{\mu}\right\}$. From the system of equations $(1-3)$ it will be shown that the region is positively invariant.

To proof this consider the following steps:

From the system of equations $(1-3)$, the total population is given by $\mathrm{N}=\mathrm{S}+\mathrm{I}+\mathrm{R}$. Therefore, adding the differential equations (1) - (3), the results becomes

$$
\begin{aligned}
\frac{d N}{d t}=\frac{d S}{d t} & +\frac{d I}{d t}+\frac{d R}{d t} \\
& =(-b S \mathrm{I}+\beta-\mu)+(b S \mathrm{I}-\mathfrak{a I}-\mu \mathrm{I})+(\mathfrak{a I}-\mu \mathrm{R}) \\
& =\beta-\mu S-\mu \mathrm{I}-\mu \mathrm{R} \\
& =\beta-\mu(\mathrm{S}+\mathrm{I}+\mathrm{R}) \\
& =\beta-\mu \mathrm{N}
\end{aligned}
$$

Thus $\frac{d N}{d t}=\beta-\mu \mathrm{N}, \mathrm{N}(0)=\mathrm{N}_{0}$. is a first order differential equation.

To solve the given equation put the equation in the standard form

$$
\frac{d N}{d t}+\mu N=\beta
$$

Now by computing the integrating factor $\mathrm{F}(\mathrm{t})=e^{\int p(t) d t}$. We have the following results

$$
\mathrm{N}(\mathrm{t})=\frac{\beta}{\mu}+\left(N_{o}-\frac{\beta}{\mu}\right) e^{-\mu t}
$$

At $\mathrm{t}=0, \mathrm{~N}(0)=\mathrm{N}_{0}$ and over a long period of time, the population approaches size $\frac{\beta}{\mu}$. So that as $\mathrm{t} \rightarrow \infty$ implies $\mathrm{N}(\mathrm{t}) \leq \frac{\beta}{\mu}$. The birth rate in this model $\beta=\mathrm{N} \mu$. After a long period of time the total number of individuals is constant $\mathrm{N}=\frac{\beta}{\mu}$. This implies that $\mathrm{N}_{0} \leq \mathrm{N}(\mathrm{t}) \leq \frac{\beta}{\mu}$. So, $\mathrm{N} \in\left[N_{0}, \frac{\beta}{\mu}\right]$. Therefore, $\Omega$ is boundedness.

\section{Model Analysis}

\section{Equilibrium Points}

The equilibrium points of the system can be obtained by equating the rate of changes to zero.

$$
\frac{d S}{d t}=\frac{d I}{d t}=\frac{d R}{d t}=0
$$


This gives two equilibrium points in the feasible region, the disease free equilibrium $\mathrm{E}_{0}=\left(\frac{\beta}{\mu}, 0,0\right)$ and the endemic steady state $E^{*}=\left(S^{*}, I^{*}, R^{*}\right)$.

The endemic steady state can be found by solving the system of equations

$$
\begin{aligned}
& \mathrm{S}=\frac{\mathfrak{a}+\mu}{b} \\
& \mathrm{I}=\frac{\beta-\mu S}{b S}=\frac{\beta b-\mu(\mathfrak{a}+\mu)}{b(\mathfrak{a}+\mu)} \\
& \quad \mathrm{R}=\frac{\mathfrak{a} I}{\mu}=\frac{\mathfrak{a}(\beta b-\mu(\mathfrak{a}+\mu))}{\mu b(\mathfrak{a}+\mu)}
\end{aligned}
$$

Hence, the endemic steady state $E^{*}=\left(S^{*}, I^{*}, R^{*}\right)$ with $S^{*}=\frac{\mathfrak{a}+\mu}{b}, I^{*}=\frac{\beta b-\mu(\mathfrak{a}+\mu)}{b(\mathfrak{a}+\mu)}$ and

$$
R^{*}=\frac{\mathfrak{a}(\beta b-\mu(\mathfrak{a}+\mu))}{\mu b(\mathfrak{a}+\mu)} \text { provided that } \mathrm{I}^{*}>0 \text { and } \mathrm{R}^{*}>0
$$

The Basic Reproduction Number $\mathbf{R}_{\mathbf{o}}$

In mathematical epidemiology an important concept is related to the basic reproduction number $\mathrm{R}_{0}$ as it serves as a threshold parameter that governs the spread of infectious diseases in a population. The basic reproduction number, $\mathrm{R}_{0}$ is a measure of the potential for disease spread in population. Mathematically, $\mathrm{R}_{0}$ is a threshold for stability of a disease free equilibrium and is related to the peak and final size of an epidemic. The reproductive number can provide significant insight into the transmission dynamics of a disease and can guide strategies to control its spread.

The basic reproduction number $\mathrm{R}_{0}$ has been defined as the average number of secondary infections that occur when one infective is introduced into a completely susceptible host population. These determine whether an epidemic will persist or die out. The disease persists when $\frac{d I}{d t}>0$. So, to find the basic reproduction number of the system consider when the disease begins spreading, $\mathrm{S}=\mathrm{N}$ and $\frac{d I}{d t}>0$ i.e.

$$
\begin{aligned}
\frac{d I}{d t}=b S I-\mathfrak{a}-\mu \mathrm{I}>0 \\
\text { Hence, } b S-\mathfrak{a}-\mu>0 \\
\mathrm{bS}>\mathfrak{a}+\mu \\
\frac{b S}{\mathfrak{a}+\mu}>\frac{\mathfrak{a}+\mu}{\mathfrak{a}+\mu}=1 \\
\mathrm{R}_{0}=\frac{b S}{\mathfrak{a}+\mu}>1
\end{aligned}
$$




$$
\mathrm{R}_{0}=\frac{b \beta}{\mu(\mathfrak{a}+\mu)}>1
$$

Thus, the basic reproduction number is $\mathrm{R}_{0}=\frac{b \beta}{\mu(\mathfrak{a}+\mu)}$.

\section{Stability Analysis}

\section{Local Stability Analysis}

We discuss the local stability of an endemic equilibrium and a disease free equilibrium points of the system of equations $(1-3)$. The disease free equilibrium point $\mathrm{E}_{0}$ is given by $\mathrm{E}_{0}=\left(\frac{\beta}{\mu}, 0,0\right)$.

Theorem: - If $\mathrm{R}_{0}<1$, then the disease free equilibrium $\mathrm{E}_{0}$ is locally asymptotically stable.

Proof: To determine the stability of the disease free equilibrium point, we turn to the Jacobean matrix of the system evaluated at the disease free equilibrium point. The Jacobean matrix at the disease free equilibrium point is given by

$$
\mathbf{J}\left(\frac{\beta}{\mu}, 0,0\right)=\left[\begin{array}{ccc}
-\mu & \frac{-b \beta}{\mu} & 0 \\
0 & \frac{b \beta}{\mu}-\mathfrak{a}-\mu & 0 \\
0 & \mathfrak{a} & -\mu
\end{array}\right]
$$

The characteristic equation of the Jacobean matrix at the disease free equilibrium point is given by

$$
(-\mu-\lambda)^{2}\left(\frac{b \beta}{\mu}-\mathfrak{a}-\mu-\lambda\right)=0
$$

The eigenvalue of the Jacobean matrix are $\lambda_{1}=-\mu, \lambda_{2}=-\mu$, and $\lambda_{3}=\frac{b \beta}{\mu}-\mathfrak{a}-\mu$. Since having all eigenvalues negative guarantees local asymptotic stability, then we need $\frac{b \beta}{\mu}<a+\mu$ to guarantee local asymptotic stability of the disease free state.

All the eigenvalues being negative means that the disease free equilibrium is asymptotically stable.

Hence, the disease free equilibrium $\left(\frac{\beta}{\mu}, 0,0\right)$ is locally asymptotically stable provided that $\mathrm{R}_{0}<1$. 


\section{Global Stability Analysis}

\section{Global Stability of Endemic Equilibrium}

We now study the properties of the endemic equilibrium points and derive the stability condition for this equilibrium point. The globally asymptotic stability of the endemic steady state is proved by constructing a Lyapunov function.

Theorem: If $\mathrm{R}_{0}>1$, then the endemic equilibrium point of $E^{*}$ is globally asymptotically stable in the interior of $\Omega$.

Proof: For the global stability of the endemic equilibrium point $\mathrm{E}^{*}$ we construct the Lyapunov function $\mathrm{L}: \Omega_{+} \rightarrow$ $R$, where $\Omega_{+}=\{S, I \in \Omega: S>0, I>0\}$ given by

$\mathrm{L}(\mathrm{S}, \mathrm{I})=\mathrm{W}_{1}\left[\mathrm{~S}-S^{*} \ln \left(\frac{S}{S^{*}}\right)\right]+\mathrm{W}_{2}\left[\mathrm{I}-\mathrm{I}^{*} \ln \left(\frac{\mathrm{I}}{\mathrm{I}^{*}}\right)\right]$

Where $\mathrm{W}_{1}$ and $\mathrm{W}_{2}$ are positive constants to be chosen letters. Take the derivative of the above function.

$$
\begin{aligned}
\frac{d L}{d t} & =\frac{d L}{d S} \frac{d S}{d t}+\frac{d L}{d I} \frac{d I}{d t} \\
= & W_{1}\left[\dot{S}_{-} S^{*}\left(\frac{S^{*}}{S}\right)\left(\frac{1}{S^{*}}\right) \dot{S}\right]+W_{2}\left[\dot{I}-I^{*}\left(\frac{I^{*}}{I}\right)\left(\frac{1}{I^{*}}\right) \dot{I}\right] \\
& =W_{1}\left[\dot{S}-\left(\frac{S^{*}}{S}\right) \dot{S}+W_{2}\left[\dot{I}-\left(\frac{I^{*}}{I}\right) \dot{I}\right]\right. \\
& =W_{1}\left[\left(\frac{S-S^{*}}{S}\right) \dot{S}\right]+W_{2}\left[\left(\frac{I-I^{*}}{I}\right) \dot{I}\right] \\
& =W_{1}\left[\left(\frac{S-S^{*}}{S}\right)(-b S I+\beta-\mu S)\right]+W_{2}\left[\left(\frac{I-I^{*}}{I}\right)(b S I-a I-\mu I)\right] \\
\frac{d L}{d t}= & W_{1}\left[\left(\frac{S-S^{*}}{S}\right)\left(-\beta+\mu S^{*}+\beta-\mu S\right)\right]+W_{2}\left[\left(\frac{I-I^{*}}{I}\right)\left(\mu I^{*}-\mu I\right)\right] \\
= & W_{1}\left[\left(\frac{S-S^{*}}{S}\right)\left(\mu S^{*}-\mu S\right)\right]+W_{2}\left[\left(\frac{I-I^{*}}{I}\right)\left(\mu I^{*}-\mu I\right)\right] \\
= & W_{1}\left[\left(\frac{S-S^{*}}{S}\right)\left(\mu\left(S^{*}-S\right)\right)\right]+W_{2}\left[\left(\frac{I-I^{*}}{I}\right)\left(\mu\left(I^{*}-I\right)\right)\right] \\
= & \frac{W_{1}}{S}\left(S-S^{*}\right)^{2} \mu-\frac{W_{2}}{I}\left(I-I^{*}\right)^{2} \mu<0
\end{aligned}
$$

Hence, the endemic equilibrium point $E^{*}$ is globally asymptotically stable in the interior of $\Omega$. 


\section{NUMERICAL SIMULATIONS}

\section{Numerical Simulations of Model}

In this section, we present the numerical simulation of the proposed model by using MATLAB. In our simulation, the susceptible individuals (S) are shown by blue color, the infective individuals (I) by green, the recovered individuals $(\mathrm{R})$ by red and vaccinated individuals $(\mathrm{V})$ by black, with time measured in days.

We choose the parameter values as follows: In figure 4.1 , we took $\beta=0.0345$,

$\mu=0.0000203, \mathfrak{a}=\frac{1}{30}, \mathrm{~b}=0.001$ and $\rho=0.9$ for the graph 4.2 , we took $\mathrm{b}=0.0004$ and $\rho=0.7$.

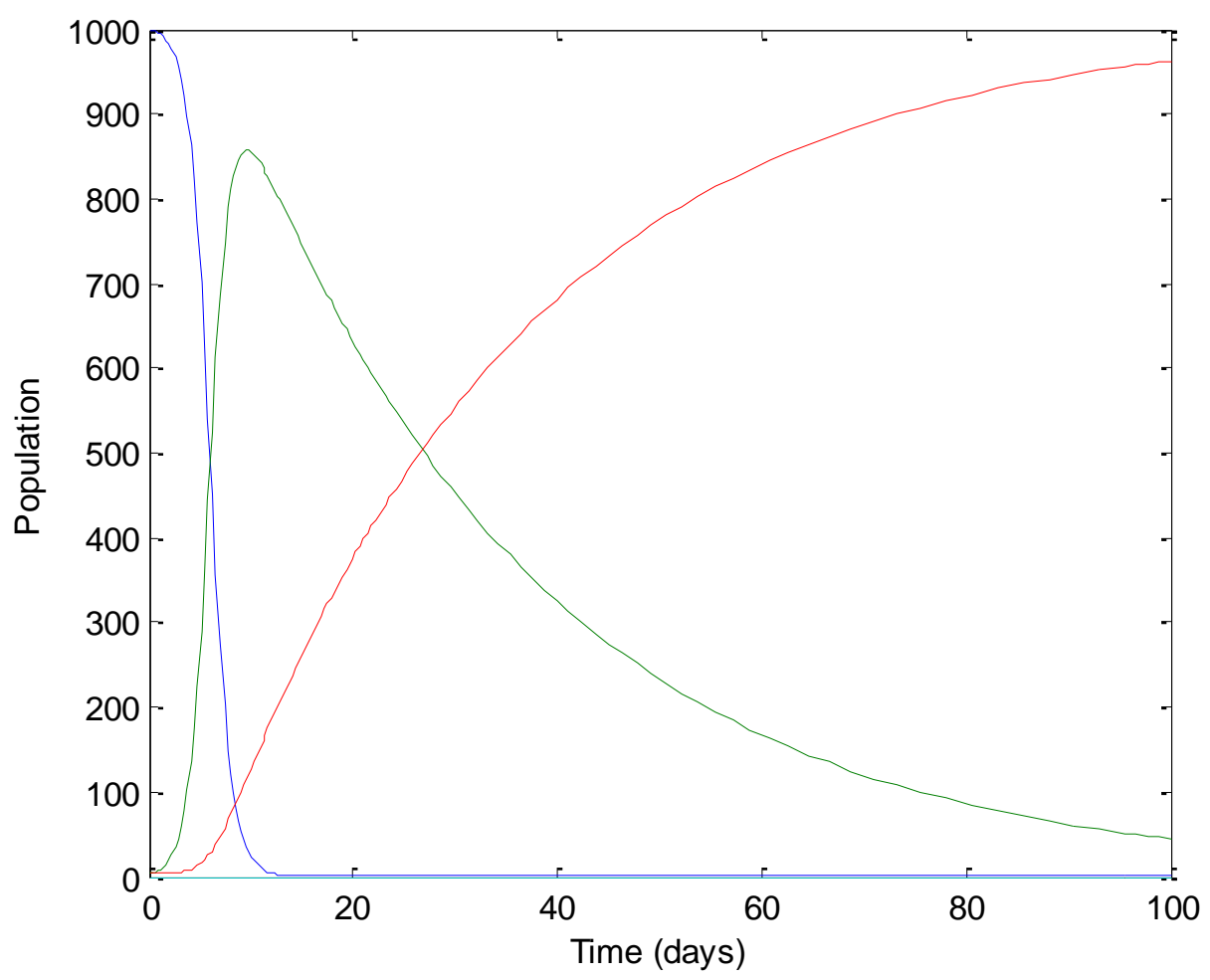

Figure 4.1: Simulation of Population 


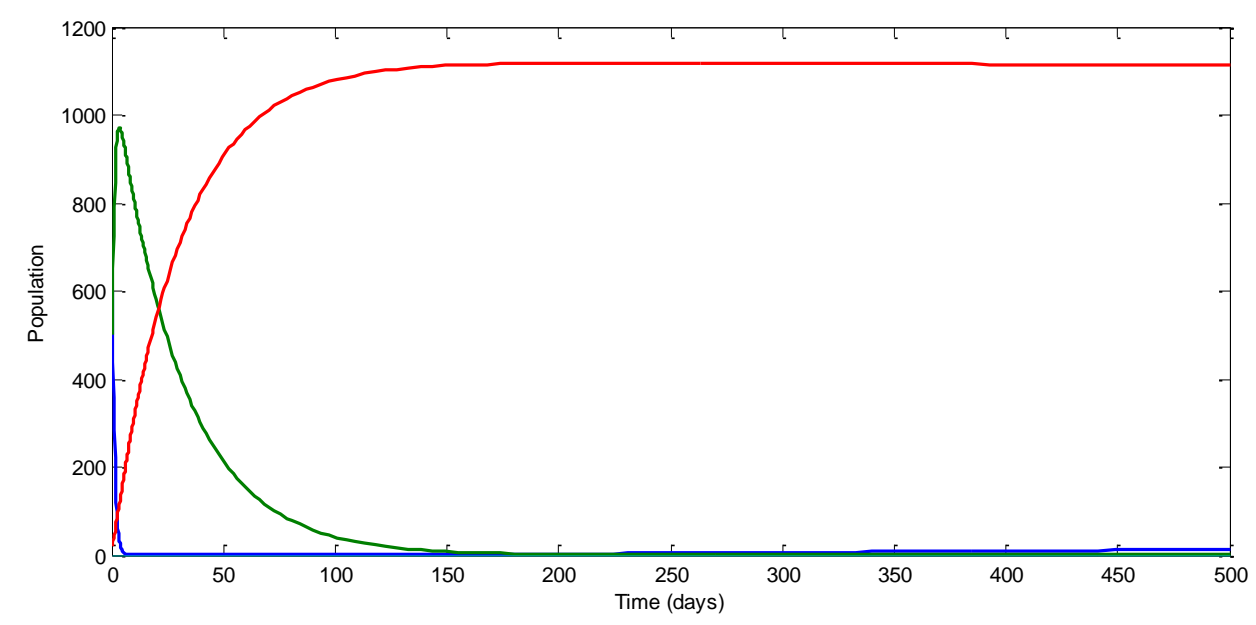

Figure 4.2: Simulation of Population

Figures 4.1 and 4.2 are showing the dynamics of the population. The susceptible population decreases as time increases. This decrease may be possibly because of the high rate of recovery due to mass vaccination, since individual become permanently immune upon recovery. The contact rate also has large impact on the spread of a disease through a population.

The higher the rates of contact, the more rapid the spread of the disease, it is also observed that as the contact rate decreases, the fraction of individuals infected decreases, the fraction of individuals infected decreases at a faster rate as would be expected logically.

We observed that that the populations of infected individuals at the very beginning rise sharply as the rate increases and the fall uniformly as time increases. This rapid decline of the infected individuals may be due to detection of the infectious disease. This graph also demonstrates that the contact rate has large impact on the spread of the disease through population. If the contact rate is observed to be high then the rate of infection of the disease will also be high as would be expected logically.

It is realized that the number of individuals Recovered rise steadily as rate increases. This may be due to early detection of the disease as well as education about the disease transmission. It can also be observed that the population of the recovered individuals rise up steadily for some number of days and then drops and remains nearly a constant. This could be due to the greater number of infectious individuals who have been treated and also acquired education about the dynamics of infectious disease transmission.

\section{Mass Vaccination}

If a vaccination programme causes the proportion of immune individuals in a population to exceed the critical threshold for a significant length of time, the transmission of the infectious disease in that population will gradually decrease. From figure 4.4 the immediate effect of the vaccine is that the peak number of infections is 
significantly reduced. Thus, the vaccine has actually not only helped those who received all of the doses of the vaccine, but some that did not. Ideally, the vaccine is given to enough individuals to completely destroy the disease, which does not need to be the entire population, a concept referred to as herd immunity. Here, herd immunity may occur due to enough susceptibles receiving partial immunity from receiving some of the doses of the vaccine.
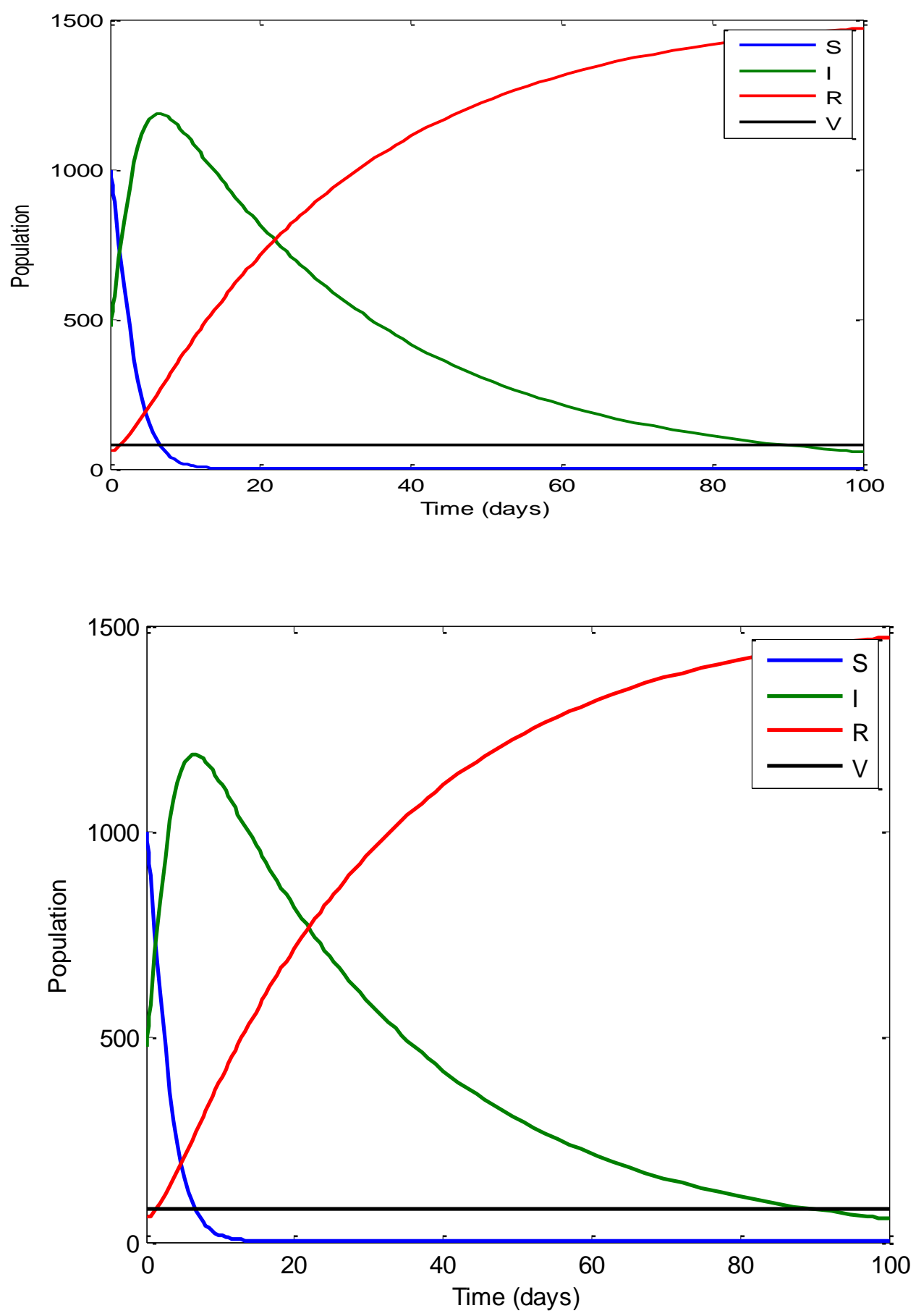

Figure 4.3 Shows disease spread with Vaccine

we choose the parameter values as: $\beta=0.0345, \mu=0.0000203, b=0.008, \mathfrak{a}=0.2$, and $\rho=0.5$. 
In the period of vaccination we consider three cases: In the first case,we say that a vaccine would be effective if the administration of a vaccine results in the number of infections changing from an increasing number to a decreasing number once the final dose of the vaccine is administered. We would say that a vaccine would be ineffective if the number of infections is still increasing after the final dose of the vaccine is administered, and a vaccine would be unnecessary if the number of infections is already decreasing before the final dose of the vaccine is administered.

Generally, an effective vaccine was chosen based on our assumption that, if a vaccine is effective, its administration should cause the number of infections to go from increasing before the final dose of the vaccine to decreasing after the final dose of the vaccine.

\section{DISCUSSION AND CONCLUSION}

\section{DISCUSSION}

Infectious diseases continue to have a major impact on individuals, populations and the economy, even though some of them have been eradicated. The spread of infectious diseases crucially depends on the pattern of contacts between individuals. Mathematical model has long been an important tool for understanding and controlling the spread of infectious diseases. Infectious disease models help us not only understand the dynamics of spreading pathogens but also design effective strategies for controlling outbreaks.

The main objective of this thesis is to develop a mathematical model which describes the dynamics of infectious disease and its transmission. In this study, an epidemic model is presented and analyzed to that effect. The basic reproductive number $\mathrm{R}_{0}$ has been computed to determine the stability of the disease. The biological meaning of the reproductive number is the average number of secondary cases produced by one infected individual during the infected individual's entire infectious period when the disease is first introduced. It characterizes the threshold behavior such that if $\mathrm{R}_{0}<1$, the modeled disease will die out if a small number of infected individuals are introduced into a susceptible population, and if $\mathrm{R}_{0}>1$, the disease will spread in the population. A good estimate of the reproductive number can provide significant insight into the transmission dynamics of the disease and can lead to effective strategies to control and eventually eradicate the disease. The basic reproductive number $\mathrm{R}_{0}$, can also be used to establish effective vaccination program. Effects of different vaccination programs on $\mathrm{R}_{0}$ are useful in setting the programs. So, we can say that reproductive ratios turned out to be an important factor in determining targets for vaccination coverage.

It was also realized that, in the absence of mass vaccination programme as well as early detection and supervised treatment, the transmission of the disease cannot be eradicated from the population. The introduction of proper and education about the infectious disease transmission as well as early detection of the disease can help reduce the disease in a population.

The results has also shown that the domain $\Omega$ is positively invariant, because no solution paths leave through any boundary. Based on results for the SIR models, we expect that all paths in $\Omega$ with infectives go to the endemic 
equilibrium if $\mathrm{R}_{0}>1$. Then we have the usual behavior for an endemic model, in the sense that the disease dies out below the threshold, and the disease goes to a unique endemic equilibrium above the threshold.

\section{CONCLUSION}

Epidemic modeling is an increasingly important tool in the study of the infectious diseases. In the SIR model, the total population is divided into three classes, susceptible, infected and recovered. The model has two equilibrium states; an infection free equilibrium and endemic equilibrium state. Many models for the spread of infectious diseases in population have been analyzed mathematically and applied to specific diseases.

Many diseases that are possible to be vaccinated against only require one dose of that vaccine, and then most of those people are immune to that disease. However, some diseases, such as Hepatitis B, require multiple doses of a vaccine, spread apart by a designated period of time, for immunity. Hepatitis B requires three doses of a vaccine, with the second occurring about 1 month after the first and the third occurring about 5 months after the second, for immunity. Thus, we wanted to look at how a multistage vaccine could work at providing population herd immunity. Specifically, we set up a model based on the classic SIR model from Kermack and McKendrick and then studied the effects of a multistage vaccine.

We found that a vaccine could be effective, ineffective, or unnecessary, depending on whether the vaccine caused a decrease in the number of infected individuals or not. The effect of a grace period on a vaccine is important. It allows for more individuals to be fully vaccinated and thus immune, potentially increasing the effectiveness of the vaccine if a sufficiently long grace period can be provided.

We were able to derive a necessary condition for a vaccine to be effective based only on parameter values as well as a test condition for effectiveness should one want to, solve the system numerically. The model has shown success in attempting to predict the causes of infectious disease transmission within a population. The model strongly indicated that the spread of a disease largely depend on the contact rates with infected individuals within a population.

In this study, however, we found that one administration of the vaccine is not enough. The disease will eventually resume whatever behavior it would have had without the vaccine. Thus, if the disease would become endemic in the population without a vaccine, it would also do so once the vaccine administration was complete. So, we then found that, pending the birth and death rates were biologically reasonable in value, and the first administration of a vaccine was effective, it would be possible to continually cause the number of infections to decrease by repeating the administration of the vaccine. This could then suppress the disease for as long as vaccines were administered and potentially for many time units after they were ceased. If a multistage vaccine is required, it should be continued to be administered for as long as the disease needs to be suppressed, which will effectively result in herd immunity for the population for a long time after the vaccines are ceased.

One of the changes we have considered is allowing for the proportions of individuals that get vaccinated to 
change based on how the first administration of the vaccine goes. If it is effective, then we would increase the proportions of people who get vaccinated in the next administration, but, if it is ineffective or unnecessary, then we would decrease the proportions of people receiving the vaccination.

Another change is to make the model more individual based, where, instead of vaccinating individuals uniformly throughout a grace period, we would allow individuals to get vaccinated at any time during that grace period, if they choose to do so. This would be much more akin to what happens in reality, so the hope is that, upon implementing this change, we would still see that a multistage vaccine could be effective for a population.

If the proportion of the population that is immune exceeds the herd immunity level for the disease, and then the disease can no longer persist in the population. Thus if this level can be exceeded by mass vaccination, then the disease can be eliminated. The model also pointed out that early detection has a positive impact on the reduction of infectious disease. People should be educated in order to create awareness to the disease transmission so that society will be aware of this deadly disease.

\section{References}

Anderson, R. M. and May, R. M. (1991), Infectious Diseases of Humans. Oxford, Oxford University Press.

Bailey, N. T. J. (1975), The Mathematical Theory of Infectious Diseases and Its Applications Griffin.

Brauer F. \& Castillo-Chavez, C. (2001). Mathematical Models in Population Biology and Epidemiology. Springer, New York.

B. T. Grenfell and A. P. Dobson,eds, (1995); Ecology of Infectious Diseases in Natural Populations, Publications of the Newton Institute, Cambridge University Press, Cambridge, England.

Busenberg, S. and K. L. Cooke (1993); Vertically Transmitted Diseases, Models and Dynamics, Biomathematics 23, Springer-Verlag, Berlin-Heidelberg-New York.

C. T. Bauch, J. O. Lloyd-Smith, M. Coffee, and A. P. Galvani (2005), Dynamically modeling SARS and respiratory EIDs, past, present, future, Epidemiology (in review).

D. Anderson and R. Watson (1980); On the Spread of a Disease with Gamma-Distributed Latent and Infectious Periods, Biometrika 67.

Daley, D.J \&Gani, J. (2005); Epidemic Modeling, An Introduction. NY, Cambridge University Press.

Dawood F. (2012); Estimated global mortality associated with the first 12 months of 2009 pandemic Influenza a H1N1 virus circulation, a modeling study. Lancet Infect Dis 12(9).

Diekmann, O., J. A. P. Heesterbeek (2000); Mathematical epidemiology of infectious disease, Model building, analysis and interpretation, John Wiley \& Sons, New York. 
Demirci, E., \& Unal, A.(2011), “ a fractional order SEIR model with density dependent death rate”. Hacettepe Journal of Mathematics and Statistics Volume 40 (2).

Fong, I. \&Alibek, K., Eds (2009), Bioterrorism and Infectious Agents, A New Dilemma for the $21^{\text {st }}$ Century, 1 edn, Springer.

Guihua L., Wendi W., and Zhen J. (2005), “ Global stability of an SEIR epidemic model with constant immigration”. Chaos, Solitons and Fractals 30 (2006) 1012 - 1019.

G. L. Mandell, R. G. Douglas, and J. E. Bennett (1979), Principles and Practice of Infectious Diseases, A Wiley Medical Publication John Wiley and Sons, New York, NY, USA.

H. M. Taylor and S. Karlin (1998), An Introduction to Stochastic Modeling, third ed., Academic Press, San Diego.

H. R. Thieme (2003), Mathematics in Population Biology, Princeton University Press, Princeton, New Jersey.

Harvigsen, G. and Pedersen, S. (2012), Lessons learned from 20 years with telemedicine in North Norway.

J. P. LaSalle (1976), The Stability of Dynamical Systems, SIAM, Philadelphia, PA.

J. Wu and Y. Luo (2004), Infectious Diseases, Central University Press, Changsha, China.

Kaufman, J., Edlund, S. and Douglas, J. (2009), 'Infectious Disease Modeling, Creating a Community to Biological Threats', Statistical Communications in Infectious Diseases 1(1).

Keeling, M.J. \&Rohani, P. (2008). "Modeling Infectious Diseases in Humans and Animals", Princeton, Princeton University Press. 